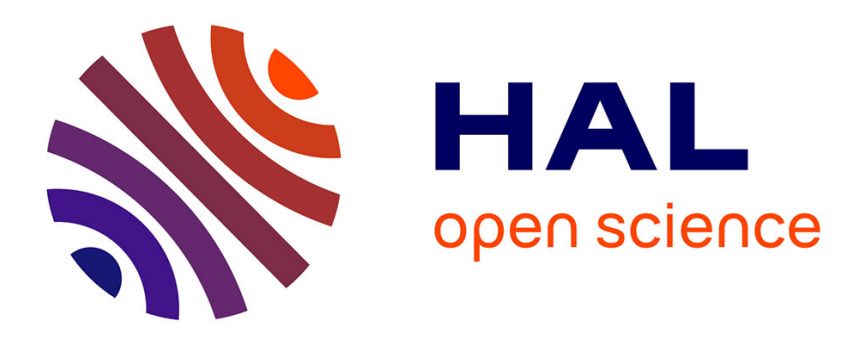

\title{
Proportional Dynamic Consensus in Open Multi-Agent Systems
}

Mauro Franceschelli, Paolo Frasca

\section{To cite this version:}

Mauro Franceschelli, Paolo Frasca. Proportional Dynamic Consensus in Open Multi-Agent Systems. CDC 2018 - 57th IEEE Conference on Decision and Control, Dec 2018, Miami, FL, United States. pp.900-905, 10.1109/CDC.2018.8619639 . hal-01945840

\section{HAL Id: hal-01945840 \\ https://hal.science/hal-01945840}

Submitted on 5 Dec 2018

HAL is a multi-disciplinary open access archive for the deposit and dissemination of scientific research documents, whether they are published or not. The documents may come from teaching and research institutions in France or abroad, or from public or private research centers.
L'archive ouverte pluridisciplinaire HAL, est destinée au dépôt et à la diffusion de documents scientifiques de niveau recherche, publiés ou non, émanant des établissements d'enseignement et de recherche français ou étrangers, des laboratoires publics ou privés. 


\title{
Proportional Dynamic Consensus in Open Multi-Agent Systems
}

\author{
Mauro Franceschelli $i^{\ddagger}$ and Paolo Frasca ${ }^{\dagger}$
}

\begin{abstract}
In this paper we investigate a dynamic consensus problem for an open multi-agent system. Open multi-agent systems are characterized by a time-varying set of agents connected by a network: agents may leave and new agents may join the network at any time, thus the term "open". The dynamic consensus problem consists in achieving agreement about the time-varying average of a set of reference signals that are assumed to be the agents' inputs. Dynamic consensus has recently found application in the context of distributed estimation for electric demand-side management, where a large population of connected domestic appliances needs to estimate its future average power consumption. Since the considered network of devices changes as new appliances log in and out, there is a need to develop and characterize dynamic consensus algorithms for these open scenarios. In this paper we give several initial contributions both to a general theory of open multi-agent systems and to the specific problem of dynamic consensus within this context. On the theoretical side, we propose a formal definition of open multi-agent system, a suitable notion of stability, and some sufficient conditions to establish it. On the applied side, we design a novel dynamic consensus algorithm, the Open Proportional Dynamic Consensus algorithm. We characterize some of its convergence properties in the proposed open-multi-agent systems framework and we illustrate its evolution by numerical simulations.
\end{abstract}

\section{INTRODUCTION}

A multi-agent system is a dynamical model for the behavior of a possibly large group of agents, e.g., robots, devices, sensors, oscillators etc., whose pattern of interactions due to sensing, communication or physical coupling is modeled by a graph that represents the network structure of the system. Most literature on multi-agent systems considers networks of fixed size, i.e., number of agents, and then considers several kinds of scenarios such as time-varying network topologies. In this paper we explicitly consider a more radical scenario of open multi-agent system where the set of agents is timevarying, i.e., agents may join and leave the network at any time.

Similar notions of "open" systems can be found in the computer science literature [1], [2] when referring to software agents and the problem of evaluating reputation in open environments. Similar open systems have also been considered in game theory [3]. Despite the abundance of works in multi-agent systems from the systems and control community, openness is rarely explicitly included in

\footnotetext{
${ }^{\ddagger}$ M. Franceschelli is with the Dept. of Electrical and Electronic Engineering, University of Cagliari, Italy, email: mauro.franceschelli@diee.unica.it.

${ }^{\dagger}$ P. Frasca is with Univ. Grenoble Alpes, CNRS, Inria, Grenoble INP, GIPSA-lab, F-38000 Grenoble, France. email: paolo.frasca@gipsa-lab.fr.

$\ddagger$ This work was supported by the Italian Ministry of Research and Education (MIUR) under call SIR 2014 with the grant "CoNetDomeSys", code RBSI14OF6H, and by Regione Autonoma della Sardegna with the 2016/2017 "Visiting Professor Program".
}

a rigorous analysis, but rather explored by simulations as in [4]. Indeed, openness implies some conceptual difficulties in adapting control-theoretic notions such as state or stability. For this reason, some authors have recently proposed to circumvent the mathematical hurdles by embedding the timevarying agent set in a time-invariant finite superset [5]. In a different perspective, others have aimed to describe the open system through significant statistical properties: encouraging results have been presented in [6], where the authors study a max-consensus problem in open multi-agent systems, and in [7], [8], where the authors study the problem of averageconsensus by gossiping.

Our attempt to formalize open dynamical systems is motivated by modern power networks, where smart appliances that are endowed with communication and computation capabilities can arrive or depart at any time. In these applications, the appliances aim to estimate in a distributed fashion the current and future power consumption of the network they belong to: this information is then used locally to optimize the power consumption of the appliances [9], [10]. The use of dynamic consensus algorithms for distributed estimation is thus a key enabler for the development of distributed control protocols among appliances that do not require centralized supervision nor centralized data aggregation that could violate the privacy of the users. Lead by this application, we concentrate on one specific kind of open multi-agent dynamics, which generalizes to the open context the wellknow Proportional Dynamic Consensus dynamics. In this dynamics, each of the nodes receives an input signal and is tasked to track the average of all inputs over the network. In the smart grid scenario, the object of the distributed estimation is the time-varying average power consumption by the network. Thus, by considering the planned power consumption of each device as an external reference signal for each agent, a dynamic consensus algorithm can be used to estimate the time-varying average value of this potentially large set of reference signals. Since devices login and logout from the network without notice, the set of reference signals is, in general, time-varying.

The dynamic consensus problem has received significant attention, as demonstrated by the forthcoming tutorial [11]. Since the early work in [12], a fundamental idea to render consensus protocols "dynamic" has been adding the derivative of each agents' own reference signal to a consensus filter that would thus track the time-varying average of the references. Several algorithms that exploit this mechanism have been proposed [4], [13], [14]: their main advantages are convergence speed and accuracy (which can be perfect for constant reference signals), while their main drawback is 
their lack of robustness with respect to errors in their initialization and, consequently, with respect to changes in the network composition. If the number of agents changes, these algorithms accumulate estimation errors that can severely deteriorate the estimation performance. Some algorithms, for instance those in [15] and [16], [17], [18], have instead shown superior robustness properties that can be useful to allow for the addition or removal of agents, even though their analysis has been so far limited to networks of fixed size. We take note that the main drawback of proportional dynamic consensus, as illustrated in [16], consists in a tradeoff between steady-state error for constant reference signals, tracking error and convergence rate. In the recent work [19], the authors propose and characterize the so-called multi-stage dynamic consensus algorithm, which consists in a cascade of proportional consensus filters and has been proved to guarantee small steady-state and tracking error for a given convergence rate, thanks to exchanging a larger quantity of local information between the agents. Furthermore, the strategy proposed in [19] has been shown to be implementable with asynchronous and randomized (gossip-based) local interactions.

a) Contribution: The main contributions of this paper are: i) new definitions of equilibrium and stability, suitable for open-multi-agent systems, together with a sufficient condition to assess such stability; ii) a modified proportional dynamic consensus algorithm, presented in discrete time, which has been augmented with a mechanism to deal with agents joining and leaving the network: we thus refer to it as the Open Proportional Dynamic Consensus algorithm. The OPDC is the first dynamic consensus algorithms that is designed for open multi-agent systems.

b) Paper structure: In Section II we introduce both our general model of open multi-agent system and the specific Open Proportional Dynamic Consensus algorithm: the latter is a modified version of the proportional dynamic consensus algorithm, suitable to be executed by an open multi-agent system. Section III proposes the new definition of stability for the considered open multi-agent system, together with some sufficient conditions. These tools are then applied to the Open Proportional Dynamic Consensus algorithm. In Section IV the case study of distributed estimation in electric demand side management is presented to show a real case of application and numerical simulations are shown to corroborate the theoretical analysis. Finally, in Section V concluding remarks are given.

\section{OPEN DYNAMICAL SYSTEMS}

For all $t \in \mathbb{Z}_{>0}$, let $G(t)=(V(t), E(t))$ be a timevarying directed graph with time-varying set of agents $V(t)$ and time-varying set of edges $E(t) \subseteq(V(t) \times V(t))$. Set $V(t)$ contains the labels corresponding to the agents that are active at time $t$ : we may think of $V(t)$ as a subset of $\mathcal{U}$, an infinite universe set of possible labels. The cardinality of set $V(t)$, that is, the number of agents that belong to the network at time $t$, is denoted as $n(t)=|V(t)|$. Two agents $i$ and $j$ are said to be neighbors at time $t$ if they share an edge at time $t$, i.e., $(i, j) \in E(t)$. Let $N_{i}(t)$ be the set of neighbors of node $i$ at time $t$, i.e., $N_{i}(t)=\{j \in V(t):(i, j) \in E(t)\}$.

For each time $t$ and each agent $v \in V(t)$, we associate a "state" variable $x_{v}(t) \in \mathbb{R}$ and an "input" variable $u_{v}(t) \in \mathbb{R}$. Note that these variables are defined only at time instants such that $v \in V(t)$. With these ingredients we can define laws that describe how the sequence $[x(t)]_{t \in \mathbb{Z}_{\geq 0}}$ evolves. Note that we will not be able to write $x(t+1)$ as a function of $x(t)$, so that the evolution of $x(t)$ does not constitute a "closed" dynamical system. Instead, we shall take as given the sequences $[V(t)]_{t \in \mathbb{Z}_{>0}},[E(t)]_{t \in \mathbb{Z}>0}$, and $[u(t)]_{t \in \mathbb{Z}_{>0}}$ (with the consistency conditions that $\bar{E}(t) \subseteq$ $(V(t) \times V(t))$ and $u(t) \in \mathbb{R}^{V(t)}$ for all $\left.t\right)$ and define the evolution law of $x$ by

$$
x(t+1)=f(x(t), V(t), V(t+1), E(t), E(t+1), u(t)) .
$$

Such update rule $f$ should distinguish three kinds of nodes $v$, respectively belonging to the sets:

- $R(t)=V(t) \cap V(t+1)$, i.e., remaining nodes that that belong both to $V(t)$ and to $V(t+1)$;

- $D(t)=V(t) \backslash V(t+1)$, i.e., departing nodes that belong to $V(t)$ but not to $V(t+1)$;

- $J(t)=V(t+1) \backslash V(t)$, i.e., joining nodes that belong to $V(t+1)$ but not to $V(t)$.

Since all $x(s)$ takes values in $\mathbb{R}^{V(s)}$, the components corresponding to $D(t)$ are simply left out from $x(t+1)$. Instead, components in $J(t)$ need to be "initialized" according to some rule. Finally, for all $v \in R(t)$ there shall be a causal evolution law in the form

$$
x_{v}(t+1)=f_{v}(x(t), V(t), E(t), u(t)) .
$$

For concreteness, we now describe one such map $f$ which we call the Open Proportional Dynamic Consensus (OPDC) algorithm.

Definition 2.1 (Open Proportional Dynamic Consensus) Let $\varepsilon>0$ and $\alpha \in(0,1)$. At each time $t \in \mathbb{Z}_{\geq 0}$, the generic agent $v \in V(t)$ updates its state according to the rules:

$$
\begin{aligned}
& \text { If } v \in R(t) \text {, then } \\
& \qquad \begin{aligned}
x_{v}(t+1)= & x_{v}(t)-\alpha\left(x_{v}(t)-u_{v}(t)\right) \\
& -\varepsilon \sum_{w \in N_{v}(t)}\left(x_{v}(t)-x_{w}(t)\right) .
\end{aligned}
\end{aligned}
$$

If $v \in J(t)$, then

$$
x_{v}(t+1)=\frac{1}{\left|N_{v}(t+1)\right|} \sum_{w \in N_{v}(t+1)} x_{w}(t) .
$$

Under the assumption that $V(t+1)=V(t)$, i.e., the set of agents does not change, the OPDC reduces to what is called Proportional Dynamic Consensus. Namely, it reduces to the update (2a), which can be written in matrix form as

$$
\begin{aligned}
x(t+1) & =x(t)-\alpha(x(t)-u(t))-L(t) x(t) \\
& =((1-\alpha) I-\varepsilon L(t)) x+\alpha u(t) \\
& =P(t) x(t)+\alpha u(t)
\end{aligned}
$$


where matrix $P(t)=(1-\alpha) I-\varepsilon L(t)$ in $\mathbb{R}^{V(t) \times V(t)}$ is a non-negative substochastic matrix with row sum and column sum equal to $(1-\alpha)$ and spectral radius $\rho=$ $\max \{|1-\alpha-\varepsilon \lambda|: \lambda$ is eigenvalue of $L(t)\}$. In what follows, we shall assume that for all $t$ the directed graph $G(t)$ is strongly connected and that the parameter $\varepsilon$ is small enough to ensure that the spectral radius of $P(t)$ is $1-\alpha$.

\section{STABILITY ANALYSIS}

We now define the concept of trajectory of points of interest which will be useful in the considered scenario of open multi-agent system.

Definition 3.1 (Trajectory of points of interest) Consider an open system (1). Assume that for every $t$, the equation

$$
y=f(y, V(t), V(t), E(t), E(t), u(t))
$$

has a unique solution and denote that solution as $x_{e}(t)$. Then, the sequence $\left[x_{e}(t)\right]_{t \in \mathbb{Z}_{\geq 0}}$ is called trajectory of points of interest of the open multi-agent system.

Observe that $x_{e}\left(t_{0}\right) \in \mathbb{R}^{V\left(t_{0}\right)}$ represents the hypothetical equilibrium of the dynamics followed by $x(t)$ if the three given sequences $V(t), E(t)$ and $u(t)$ would be kept constant for all $t \geq t_{0}$. Consequently, $x_{e}\left(t_{0}\right)$ is determined only by information at time $t_{0}$ : the time-variance of $V(t), E(t)$ and $u(t)$ does not imply any ambiguity in the definition of the sequence $x_{e}(t)$.

As an example, consider system (2). Under our standing assumptions on $P(t)$, the solution $x_{e}(t)$ is unique for every $t$ and can be computed as

$$
x_{e}(t)=(I-P(t))^{-1} \alpha u(t)=\left(I+\frac{\varepsilon}{\alpha} L(t)\right)^{-1} u(t) .
$$

Next, we define a so-called "open" distance function which is used to evaluate the distance between two points with labeled elements that belong to Euclidean spaces of different dimensions. In the particular case in which the two points have elements with the same labels, i.e., the same agents, the open distance function reduces to the Euclidean distance.

Definition 3.2 (Open distance function) Let $V_{1}$ and $V_{2}$ be two finite sets of node indices. Let $d: \mathbb{R}^{V_{1}} \times \mathbb{R}^{V_{2}} \rightarrow \mathbb{R}_{\geq 0}$ be defined as

$$
d(x, y)=\sqrt{\sum_{v \in V_{1} \cap V_{2}}\left(x_{v}-y_{v}\right)^{2}+\sum_{v \in V_{1} \backslash V_{2}} x_{v}^{2}+\sum_{v \in V_{2} \backslash V_{1}} y_{v}^{2}}
$$

for any $x \in \mathbb{R}^{V_{1}}$ and $y \in \mathbb{R}^{V_{2}}$.

The open distance (4) satisfies several natural properties, which we summarize in the next statement.

Proposition 3.3 (Open distance function) Function $d(x, y)$ in (4) is such that for any vectors $x, y$, and $z$ :

1) $d(x, y) \geq 0$;

2) $d(x, y)=d(y, x)$;
3) If $x=y$, then $d(x, y)=0$;

4) $d(x, z) \leq d(x, y)+d(y, z)$

Note that the converse of the third implication does not hold. Indeed, consider $x \in \mathbb{R}^{\{1,2\}}$ to be $x=[1,0]$ and $y \in$ $\mathbb{R}^{\{1\}}$ to be $[1]$. Then, $d(x, y)=0$ despite the two vectors being different.

Having this open distance available, we can naturally use it on the trajectories of points of interest.

Definition 3.4 (Trajectory of points of interest of bounded variation) A trajectory of points of interest $x_{e}(t)$ is said to have bounded variation if there exists a constant $B \geq 0$ such that $d\left(x_{e}(t+1), x_{e}(t)\right) \leq B$ for all $t \in \mathbb{Z}_{\geq 0}$ where $d(\cdot, \cdot)$ is the open distance function in (4).

The next definition introduces a notion akin to a weak form of Lyapunov stability for open multi-agent systems.

Definition 3.5 (Stability of a trajectory of points of interest) Let $x(t)$ be the evolution of an open system. A trajectory of points of interest $x_{e}(t)$ with bounded variation is said to be stable if there exists $R \geq 0$ such that for every $\varepsilon>R$, there exists $\delta>0$ such that if $d\left(x(0), x_{e}(0)\right)<\delta$ then $d\left(x(t), x_{e}(t)\right)<\varepsilon$ for every $t \geq 0$.

The next result is a very simple sufficient condition to ensure stability in the above sense.

Theorem 3.6 (Stability of Open Multi-agent Systems) Consider an open multi-agent system with state trajectory $x(t)$ and having $x_{e}(t)$ as trajectory of points of interest of bounded variation $B$. If there exist $\gamma \in(0,1)$ and $\Gamma \geq 0$ such that

$$
d\left(x(t+1), x_{e}(t+1)\right) \leq \gamma d\left(x(t), x_{e}(t)\right)+\Gamma,
$$

then the open multi-agent system is stable according to Definition 3.5 with $R \geq \frac{B+\Gamma}{1-\gamma}$.

Proof: By hypothesis, the trajectory of points of interest has bounded variations as per Definition 3.4, thus it holds $d\left(x_{e}(t+1), x_{e}(t)\right)<B$ for any $t \geq 0$. By the assumptions of the theorem, it holds

$$
\begin{aligned}
d\left(x(t+1), x_{e}(t+1)\right) \leq & d\left(x(t+1), x_{e}(t)\right) \\
& +d\left(x_{e}(t+1), x_{e}(t)\right) \\
\leq & \gamma d\left(x(t), x_{e}(t)\right)+\Gamma+B .
\end{aligned}
$$

This last quantity is smaller than $d\left(x(t), x_{e}(t)\right)$ if

$$
d\left(x(t), x_{e}(t)\right)>R \geq \frac{B+\Gamma}{1-\gamma} .
$$

We then conclude that, as long as $d\left(x(t), x_{e}(t)\right)>R$,

$$
d\left(x(t+1), x_{e}(t+1)\right)<d\left(x(t), x_{e}(t)\right) .
$$

Now, for any $\varepsilon>R$, we can simply choose $\delta<\epsilon$, so that by (5) it holds $d\left(x(t), x_{e}(t)\right)<\varepsilon$ for all $t \geq t_{0}$. This proves the stability of the considered open system according to Definition 3.5.

In order to apply this general result to OPDC algorithm, we need to make some assumptions about the impact of 
leaving and joining nodes. A simple such assumption would be the existence of $\Gamma>0$ such that for all $t$

$$
\Gamma^{2} \geq \sum_{v \in D(t)} x_{e, v}^{2}(t)+\sum_{v \in J(t)} x_{v}^{2}(t+1) .
$$

We believe that this assumption is not overly restrictive. For the OPDC algorithm, if both the input signals and the number of agents that join and leave at each instant of time are bounded, then it is easy to find such a bound $\Gamma$. For instance, consider the case in which initial conditions and input signals are bounded in the interval $[0,1]$ and the number of agents that may join or leave is limited to one at each instant of time. Then, one can choose $\Gamma=\sqrt{2}$.

Proposition 3.7 (Stability of Open Proportional Dynamic Consensus) The Open Proportional Dynamic Consensus is stable with $R=\frac{B+\Gamma}{\alpha}$ if its trajectory of points of interest has bounded variation $B$ and there exists $\Gamma>0$ that satisfies (7).

Proof: Let us define for all $t$ the vector $y(t+1)=$ $P(t) x(t)$ : note that $y(t+1) \in \mathbb{R}^{V(t)}$ and that the restrictions to $R(t)$ of $x(t+1)$ and $y(t+1)$ coincide. We then observe that

$$
\begin{aligned}
& d\left(x(t+1), x_{e}(t+1)\right) \\
& \leq d\left(x(t+1), x_{e}(t)\right)+d\left(x_{e}(t+1), x_{e}(t)\right) \\
& \leq \sqrt{\sum_{v \in R(t)}\left(x_{v}(t+1)-x_{e, v}(t)\right)^{2}} \\
& \overline{+\sum_{v \in D(t)} x_{e, v}^{2}(t)+\sum_{v \in J(t)} x_{v}^{2}(t+1)}+B \\
& \leq \sqrt{\sum_{v \in R(t) \cup D(t)}\left(y_{v}(t+1)-x_{e, v}(t)\right)^{2}} \\
& +\sqrt{\sum_{v \in D(t)} x_{e, v}^{2}(t)+\sum_{v \in J(t)} x_{v}^{2}(t+1)}+B \\
& \leq\left\|P(t) x(t)+\alpha u(t)-x_{e}(t)\right\|_{2}+\Gamma+B \\
& =\left\|P(t) x(t)-P(t) x_{e}(t)\right\|_{2}+\Gamma+B \\
& \leq\|P(t)\|_{2}\left\|x(t)-x_{e}(t)\right\|_{2}+\Gamma+B \\
& \leq(1-\alpha)\left\|x(t)-x_{e}(t)\right\|_{2}+\Gamma+B \\
& =\gamma d\left(x(t), x_{e}(t)\right)+\Gamma+B \text {, }
\end{aligned}
$$

where we used (7) and $\gamma=1-\alpha$. By assumption, we have $d\left(x_{e}(t+1), x_{e}(t)\right) \leq B$ for all $t \geq 0$ and the conditions in Theorem 3.6 are satisfied.

\section{NUMERICAL SIMULATIONS}

In this section we simulate numerically the open proportional dynamic consensus protocol with tuning parameter $\alpha=0.01$. We consider the problem of estimating the timevarying average of the power consumption by a network of smart appliances at a given time of the day. We consider a network composed at time $t=0$ by 200 agents, each with constant input $u_{i}(t)$ chosen uniformly at random in the interval $[0,1]$, representing the power consumption of device $i$ at a given hour of the day. We numerically simulate two scenarios. In the first, called Scenario A, at each instant of time $t$ one agent leaves the network with probability $p_{l}=$ 0.05 and one joins the network with probability $p_{j}=0.1$, thus resulting in a network which, in expectation, increases in size. In the second, called Scenario B, at each instant of time $t$ one agent leaves the network with probability $p_{l}=0.1$ and one joins the network with probability $p_{j}=0.1$, thus resulting in a network which, in expectation, does not change size. In both scenarios at each instant of time the network topology is that of a random graph with probability of edge existence between any two nodes equal to $p=0.1$.

We now discuss the numerical simulation of Scenario A where an agent is more likely to join than to leave. In Figure 1 it is shown the time-varying number of agents. In Figure 2 it is shown the average of the inputs $\bar{u}(t)=$ $\frac{1}{n(t)} \sum_{v \in V(t)} u_{v}(t)$, which is time-varying due to joining and leaving agents. In Figure 3 it is shown the evolution of the open distance function $d\left(x(t), x_{e}(t)\right)$, i.e., the distance between the state of the network at time $t$ and the point of interest $x_{e}$ at time $t$. Finally, in Figure 4 it is shown the evolution of $d(x(t), \bar{u}(t))$, which is a natural measure of the estimation error in dynamic consensus algorithms. From the simulations it can be seen that the distance functions are monotonically decreasing despite the time-varying number of agents up to threshold value which is determined by the join/leave dynamics of the agents.

We now discuss the numerical simulation of Scenario B where agents are equally likely to join or leave. In Figure 5 it is shown the time-varying number of agents. In Figure 6 it is shown the evolution of the open distance function $d\left(x(t), x_{e}(t)\right)$, i.e., the distance between the state of the network at time $t$ and the point of interest $x_{e}$ at time $t$. Finally, in Figure 7 it is shown the evolution of $d(x(t), \bar{u}(t))$. It can be seen that also in this scenario the distance functions are monotonically decreasing up to threshold value which is determined by join/leave dynamics of the agents.

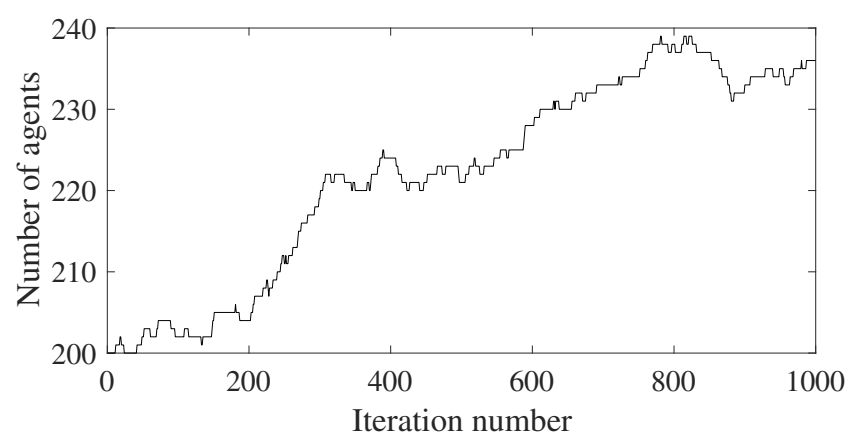

Fig. 1. Scenario A: Time-varying number of agents $|V(t)|$

\section{CONCLUSIONS}

In this paper, we have proposed a mathematical framework for open multi-agent systems, i.e., multi-agent systems that are characterized by a time-varying number of agents that join and leave the network. This generality is motivated by 


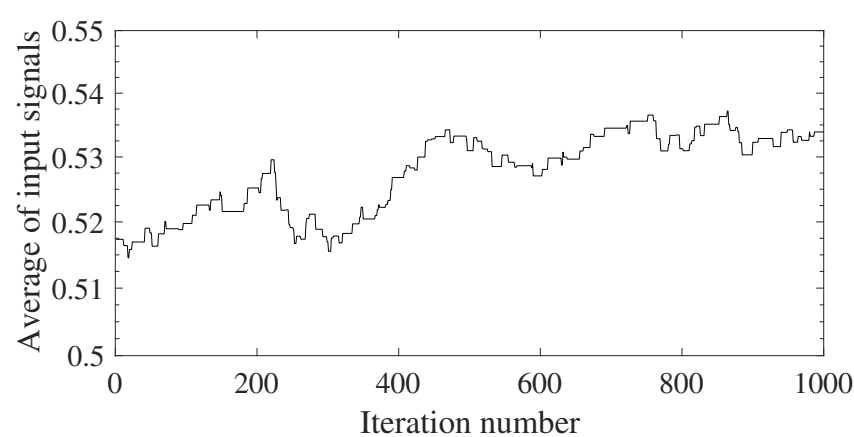

Fig. 2. Scenario A: Average of input reference signals $\bar{u}(t)$

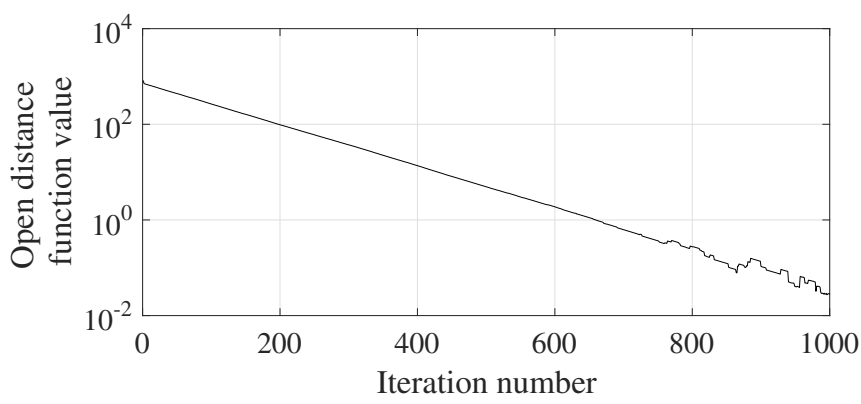

Fig. 3. Scenario A: Evolution of open distance function $d\left(x(t), x_{e}(t)\right)$.

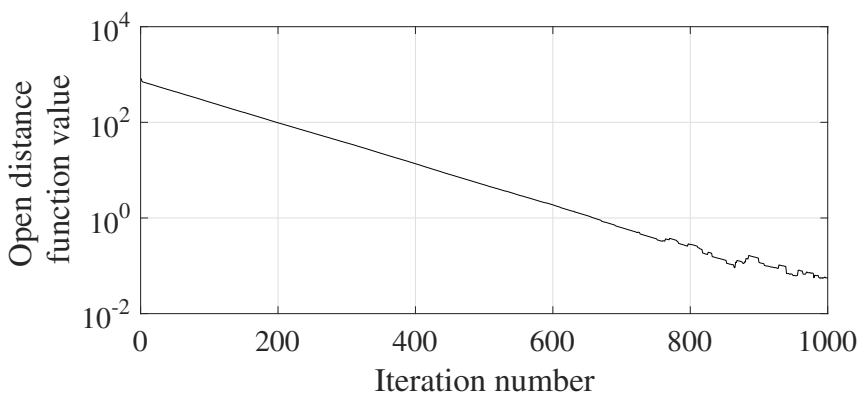

Fig. 4. Scenario A: Evolution of open distance function $d(x(t), \bar{u}(t))$ (estimation error).

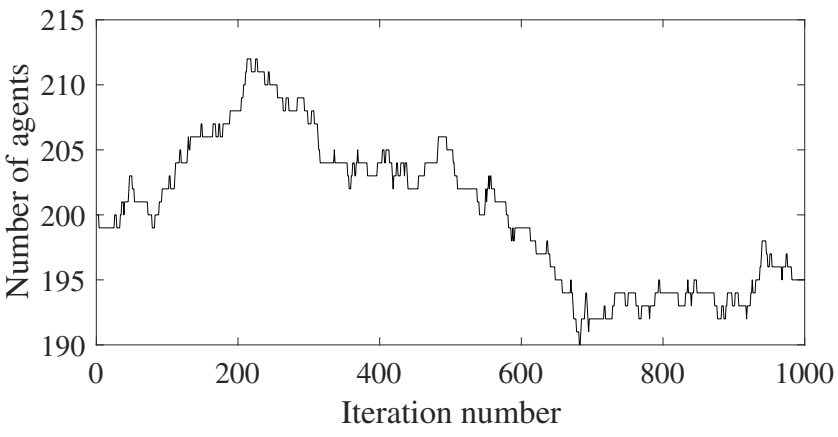

Fig. 5. Scenario B: Time-varying number of agents $|V(t)|$

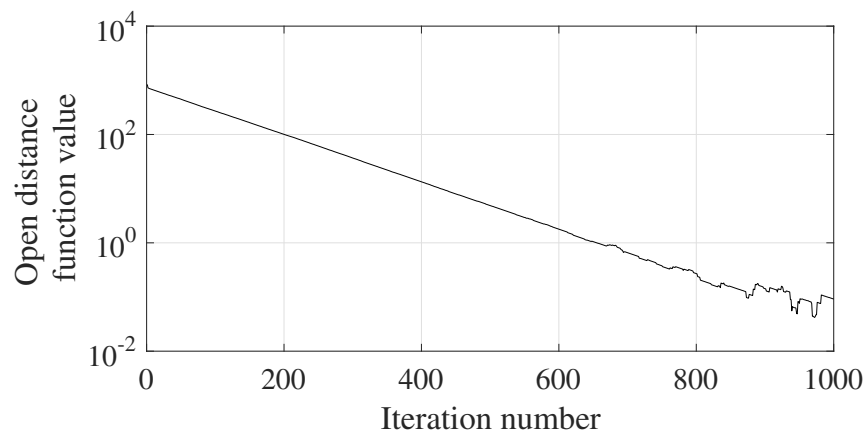

Fig. 6. Scenario B: Evolution of open distance function $d\left(x(t), x_{e}(t)\right)$.

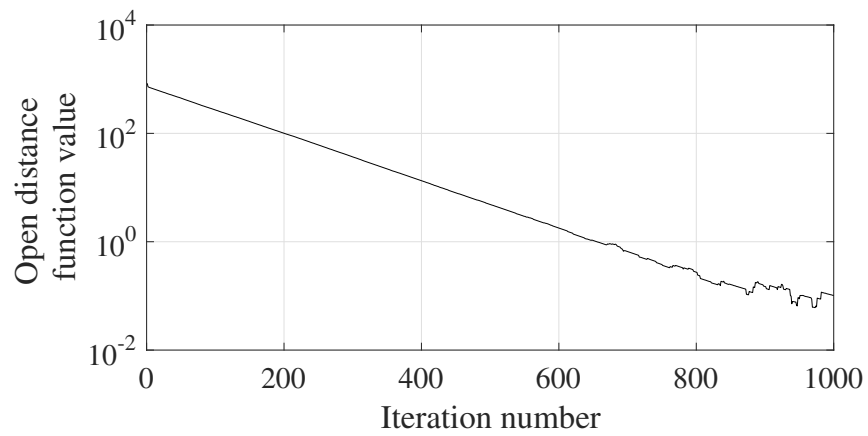

Fig. 7. Scenario B: Evolution of open distance function $d(x(t), \bar{u}(t))$ (estimation error).

a distributed estimation problem in the context of electric demand-side management, where a large population of smart appliances interconnected via a peer-to-peer network over the internet needs to estimate the average of the future planned power consumption in an environment where devices may $\log$-in and log-out without notice. Thus motivated, we proposed a novel definition of stability for open multi-agent systems and proposed a dynamic consensus algorithm, the Open Proportional Dynamic Consensus algorithm, which enables the agents to estimate in distributed fashion the timevarying average of a time-varying set of reference signals. Preliminary results on the characterization of the stability of the proposed algorithm have been provided. Numerical simulations show that the proposed algorithm is robust to time-varying number of agents.

This paper opens several research avenues. On the theoretical side, we have only scratched the surface of a possible theory of open dynamical systems. On the practical side, other dynamic consensus protocols can be envisioned and tested in concrete estimation problems.

\section{REFERENCES}

[1] T. D. Huynh, N. R. Jennings, and N. R. Shadbolt, "An integrated trust and reputation model for open multi-agent systems," Autonomous Agents and Multi-Agent Systems, vol. 13, no. 2, pp. 119-154, Sep 2006.

[2] I. Pinyol and J. Sabater-Mir, "Computational trust and reputation models for open multi-agent systems: a review," Artificial Intelligence Review, vol. 40, no. 1, pp. 1-25, Jun 2013. 
[3] D. Shah and J. Shin, "Dynamics in congestion games," in Proceedings of the ACM SIGMETRICS International Conference on Measurement and Modeling of Computer Systems-SIGMETRICS. Association for Computing Machinery/ACM-Sigmetrics, 2010.

[4] M. Zhu and S. Martínez, "Discrete-time dynamic average consensus," Automatica, vol. 46, no. 2, pp. 322 - 329, 2010.

[5] V. S. Varma, I. C. Morarescu, and D. Nesic, "Open multi-agent systems with discrete states and stochastic interactions," IEEE Control Systems Letters, vol. 2, no. 3, pp. 375-380, July 2018.

[6] M. Abdelrahim, J. M. Hendrickx, and W. P. M. H. Heemels, "Maxconsensus in open multi-agent systems with gossip interactions," in IEEE Conference on Decision and Control (CDC) 2017, Melbourne, Australia, 2017.

[7] J. M. Hendrickx and S. Martin, "Open multi-agent systems: Gossiping with deterministic arrivals and departures," in 2016 54th Annual Allerton Conference on Communication, Control, and Computing (Allerton), Sept 2016, pp. 1094-1101.

[8] — , "Open multi-agent systems: Gossiping with random arrivals and departures," in IEEE Conference on Decision and Control (CDC) 2017, Melbourne, Australia, dec 2017.

[9] M. Franceschelli, A. Pilloni, and A. Gasparri, "A heuristic approach for online distributed optimization of multi-agent networks of smart sockets and thermostatically controlled loads based on dynamic average consensus," in 17th annual European Control Conference, July 2018.

[10] M. Franceschelli, A. Gasparri, and A. Pisano, "Coordination of electric thermal systems for distributed demand-side management: A gossipbased cooperative approach," in 15th annual European Control Conference, July 2016.

[11] S. S. Kia, B. V. Scoy, J. Cortés, R. Freeman, K. Lynch, and S. Martínez, "Tutorial on dynamic average consensus: the problem, its applications, and the algorithms," IEEE Control Systems Magazine, 2018, submitted. [Online]. Available: http://carmenere.ucsd.edu/jorge/ publications/data/2018_KiScCoFrLyMa-csm.pdf

[12] D. P. Spanos, R. Olfati-Saber, and R. M. Murray, "Dynamic consensus on mobile networks," in IFAC world congress, 2005.

[13] S. Nosrati, M. Shafiee, and M. B. Menhaj, "Dynamic average consensus via nonlinear protocols," Automatica, vol. 48, no. 9, pp. $2262-$ 2270, 2012.

[14] S. S. Kia, J. Cortés, and S. Martínez, "Distributed event-triggered communication for dynamic average consensus in networked systems," Automatica, vol. 59, pp. 112 - 119, 2015.

[15] E. Montijano, J. I. Montijano, C. Sagues, and S. Martínez, "Robust discrete time dynamic average consensus," Automatica, vol. 50, no. 12, pp. $3131-3138,2014$.

[16] R. A. Freeman, P. Yang, and K. M. Lynch, "Stability and convergence properties of dynamic average consensus estimators," in Proceedings of the 45th IEEE Conference on Decision and Control, Dec 2006, pp. 338-343.

[17] H. Bai, R. A. Freeman, and K. M. Lynch, "Robust dynamic average consensus of time-varying inputs," in 49th IEEE Conference on Decision and Control, Dec 2010, pp. 3104-3109.

[18] B. V. Scoy, R. A. Freeman, and K. M. Lynch, "A fast robust nonlinear dynamic average consensus estimator in discrete time," in 5th IFAC Workshop on Distributed Estimation and Control in Networked Systems NecSys 2015, vol. 48, no. 22, 2015, pp. 191 - 196.

[19] M. Franceschelli and A. Gasparri, "Multi-stage discrete time dynamic average consensus," in 55th IEEE Conference on Decision and Control, Dec 2016. 